Article

\title{
Dental Resin-Zirconia Bonding Promotion Using High-Silica PVD Coating with High Ionization Sputtering Processing
}

\author{
Mohamed Mahmoud Abdalla ${ }^{1,2}$, Christie Ying Kei Lung ${ }^{1}$, James Kit Hon Tsoi ${ }^{1, * \mathbb{D}}$ and \\ Jukka Pekka Matinlinna ${ }^{1}$ \\ 1 Dental Materials Science, Applied Oral Sciences, Faculty of Dentistry, The University of Hong Kong, \\ Hong Kong, China; mohamabd@hku.hk (M.M.A.); cyklung@hku.hk (C.Y.K.L.); jpmat@hku.hk (J.P.M.) \\ 2 Dental Biomaterials Department, Faculty of Dental Medicine, Al Azhar University, Cairo 11751, Egypt \\ * Correspondence: jkhtsoi@hku.hk
}

Received: 19 February 2019; Accepted: 5 March 2019; Published: 11 March 2019

check for updates

\begin{abstract}
Purpose: To evaluate the effect of high-silica coating deposited by high-silica physical vapor deposition (PVD) as a chemical bonding method on resin-zirconia bond strength under different aging conditions. Methods: Twelve Y-TZP blocks were used as the substrates. Four resin cement stubs were bonded on each Y-TZP block, with a total number of 48 resin cement stubs. Two test groups $(n=24)$ were evaluated: conventional Tribochemical silica-coating (TSC) and high-silica PVD with high ionization sputtering processing. Experimental silane primer (MPS) was brushed over the surface treated Y-TZP blocks, then a polyethylene mold was placed over the coated Y-TZP blocks and filled with the adhesive resin cement, then light-cured for $40 \mathrm{~s}$. The shear bond strength (SBS) was then evaluated in dry condition and after thermo-cycling for 6000 cycles. Surface roughness, mode of failure, surface topography and elemental analysis were also evaluated. Results: In dry condition, PVD-coated zirconia specimens showed significantly higher mean SBS values $(11.7 \pm 1.3 \mathrm{MPa})$ compared to TSC $(10.2 \pm 1.1 \mathrm{MPa})(p=0.027)$. The SBS values of TSC and PVD-coated samples after thermo-cycling were higher than in dry condition, but with no statistical significant difference $(p>0.05)$. Tetragonal-to-monoclinic phase transformation was detected in TSC, but not in PVD-coated zirconia. Significant decrease in surface roughness of PVD samples compared to TSC samples $(p<0.001)$. The silica content in PVD coating was $51 \%$ as detected by EDX. Conclusions: High-silica PVD coating on zirconia can give a reliable resin-zirconia chemical bond without any phase transformation and surface destruction by conventional grit-blasting.
\end{abstract}

Keywords: physical vapor deposition (PVD); zirconia; tribochemical silica coating (TSC); shear bond strength (SBS); thermo-cycling

\section{Introduction}

Yttria-stabilized tetragonal zirconia polycrystal (Y-TZP) is a ceramic material that is used as an alternative to conventional metal-ceramic restorations due to its biocompatibility and superior mechanical properties [1-4]. These high mechanical properties are derived with a stress-induced transformation, from a meta-stable tetragonal phase to a stable monoclinic phase [5,6]. The clinical success of ceramic restorations depends not only on their strength but also on the cementation process [7]. Proper adhesive cementation to zirconia ceramics is desirable, since it improves retention, marginal adaptation, fracture resistance, durability of restorations, and enables more conservative cavity preparations $[2,8]$. However, some clinical studies have reported restoration debonding as a type of failure [9]. Unlike silica-based ceramics, which can be hydrofluoric acid (HF)-etched, followed 
by silane coupling agent application before resin cementation that yields high resin-ceramic bond strength [10-12], zirconia is inherently non silica-based, chemically inert, and resists HF treatment with the exception of high temperature HF treatment [13]. Therefore, it is difficult to achieve a strong resin bonding to zirconia with silanes [14].

Given this, various methods have been advocated to promote adhesion between the resin cement and zirconia, most commonly tribochemical silica coating (TSC), i.e., grit-blasting using silica-coated alumina grits and then application of silane, [15], chemical vapor deposition (CVD), laser irradiation [16], and selective infiltration etching $[17,18]$. However, significant variations in bond strength results in several studies have not been able to substantiate credible adhesive procedures for zirconia ceramics $[15,19]$. It has been reported that air-abrasion with $50 \mu \mathrm{m}$ alumina particles at $0.25 \mathrm{MPa}$ followed by 10-methacryloxydecyl-dihydrogenphosphate (MDP) primer is the recommended first choice of bonding to zirconia; however, air abrasion surface treatment may have an unfavorable effect on zirconia substrate, even though the bond strength is durable [20].

In principle, the application of silane coupling agents has been proven to improve the bonding between resin cement and silica-coated substrates [21]. After acid hydrolysis of silane coupling agent, it forms silanols $(-\mathrm{Si}-\mathrm{OH})$. These silanol groups, when silane is applied to surface modified zirconia, react with surface hydroxyl groups on the silica-coated zirconia to form a strong siloxane (-Si-O-Si-) linkage. When the resin cement is bonded to silanized zirconia surface, the $>C=C<$ groups in the resin cement react with the silane coupling agent. Hence, chemical bonding is formed between resin cement and zirconia [22]. This is a common method used for bonding to zirconia. However, it requires the presence of sufficient silica content on the zirconia surface for the silane to form chemical bonding [21].

TSC is the gold standard silica coating method used with these procedures; however, some studies have shown that TSC procedures cause phase transformation in Y-TZP, from tetragonal to monoclinic $(t \rightarrow m)$ [16], and induce surface flaws with a typical ploughing pattern on the surface [23]. The $t \rightarrow m$ phase transformation would lead to the dislodgement of grains, and thus form micro-cracks on its surface that can lead to catastrophic fracture of the bulk zirconia from surface flaws. In addition, the delamination of silica-coated alumina particles during ultrasonic cleaning can reduce the silica content [24]. To overcome these drawbacks, silica content coated onto the zirconia needs to be increased, so as to provide durable bond strength at the resin-zirconia interface with the aid of silane coupling agent. Moreover, achieving a reliable bond strength with zirconia using chemical bonding only, without any micromechanical roughening, is desirable, since this will reduce the potential of deterioration of the properties of zirconia substrate.

In the present study, we examine the deposition of thin silica coating using physical vapor deposition (PVD) with high ionization sputtering (arc splitting) processing. PVD is a vacuum deposition process in which the coating material is evaporated by high-energy ionized gas bombardment under vacuum, followed by vapor phase transportation to the substrate, which results in deposition of a uniform smooth surface coating $[25,26]$. The advantages of this coating method are the ability of deposition of high silica content for better bonding with silane coupling agents, high level of adhesion to substrates as a result of ions sputtering, low contamination, environment friendly, no damage to substrate surface; it was also recently found to reduce the low-temperature degradation (LTD) susceptibility of zirconia $[25,27]$. The film thickness and composition of the coating can be easily altered by manipulating the process parameters.

The aim of this laboratory study was to evaluate the effect of depositing high content of silica layer by PVD high ionization sputtering process on the bond strength of resin cement to zirconia as well as the effect of artificial aging on the bond durability, when compared with TSC. 3-methacryloxypropyltrimethoxysilane (MPS) was used to silanize the silica-coated zirconia surfaces. The null hypothesis of this study was that there is no difference in shear bond strength between the PVD sputtering method and TSC, and that thermo-cycling does not deteriorate bond strength. 


\section{Materials and Methods}

\subsection{Zirconia Surface Preparation}

Twelve Y-TZP (Upcera Dental Zirconia Blank U9810W52, Liaoning Upcera, Benxi, Liaoning, China) blocks were used as the substrates for surface treatment and resin bonding, with four resin specimens bonded on each Y-TZP block, in addition to five other blocks for surface roughness evaluation, surface topography and elemental analysis. A band saw was used to cut the Y-TZP blocks from a pre-sintered circular blank. Polishing of the zirconia blocks was done with 320-grit silicon carbide ( $\mathrm{SiC}$ ) abrasive paper under running water using a polishing machine (Lunn, $\mathrm{UK}$ ). The blocks were then cleansed ultrasonically (DFS200, Decon Ultrasonic, Hove, East Sussex, UK) in $70 \%$ ethanol (BDH Reagents \& Chemicals, Chicago, IL, USA), for $10 \mathrm{~min}$, and then air dried at room temperature. The blocks were sintered in a furnace at a rate of $3{ }^{\circ} \mathrm{C} / \mathrm{min}$ and maintained at $1480{ }^{\circ} \mathrm{C}$ for $3 \mathrm{~h}$ according to the manufacturer specifications. The final size of fully sintered Y-TZP blocks was approximately $8.0 \times 6.5 \times 39.0 \mathrm{~mm}^{3}$.

A total number of 48 resin cement specimens were evaluated for shear bond strength (SBS). They were randomly divided into two groups $(n=24)$ according to the surface treatment method used to coat the zirconia blocks: (1) surface treatment by TSC, and (2) surface treatment by PVD sputtering method. Then, each group was subdivided into two subgroups $(n=12)$ according to the aging condition; the first subgroup was kept in a desiccator at room temperature for $24 \mathrm{~h}$ prior to bond strength testing (Dry group) to obtain the initial bond strength. The second subgroup was subjected to thermo-cycling for 6000 cycles between $5.0 \pm 0.5^{\circ} \mathrm{C}$ and $55.0 \pm 0.5^{\circ} \mathrm{C}$ with $20 \mathrm{~s}$ dwell time for each in deionized water bath (Thermo-cycled group) [28].

\subsection{TSC Specimens' Preparation}

One surface of the Y-TZP blocks was tribochemically silica-coated using $110 \mu \mathrm{m}$ silica-coated alumina powder (Rocatec Plus, 3M-ESPE, Seefeld, Germany) with a sandblasting device. The operating pressure used was $300 \mathrm{kPa}$ for $15 \mathrm{~s}$ per $1 \mathrm{~cm}^{2}$ with the nozzle being in a constant perpendicular distance of $10 \mathrm{~mm}$ [29]. The Y-TZP blocks were then cleansed in an ultra-sonic bath in 70\% ethanol for $10 \mathrm{~min}$ and then rinsed with $70 \%$ ethanol.

\subsection{High Silica Content Coating by PVD-High Ionization Sputtering Technique}

The silica films were deposited onto the polished and cleansed Y-TZP blocks by using a closed-field unbalanced magnetron sputtering system (MAT, Immenhausen, Germany) with a four-target configuration. The magnetron sputtering system comprised of Si target (purity $>99.9$ at.\%). The coating equipment was furnished with a rotating substrate holder at a speed of $10 \mathrm{rpm}$ to permit a homogeneous composition of the coatings, and the target-to-substrate distance was $17 \mathrm{~cm}$. The vacuum chamber was pumped down to a background pressure less than $3 \times 10^{-6}$ Torr $(0.4 \mathrm{mPa})$ before deposition. After that, the Ar working gas (purity 99.995 at.\%) pressure was set at $\sim 1.5 \times 10^{-3}$ Torr $(200 \mathrm{mPa}$ ) by controlling the flow rate $(50 \mathrm{sccm})$ of argon. Oxygen $\left(\mathrm{O}_{2}\right)$ was used as reactive gas (flow rate 50-80 sccm) for the formation of oxide films. The total pressure of $\mathrm{Ar} / \mathrm{O}_{2}$ mixed gases during reactive sputtering was $\sim 4 \times 10^{-3}$ Torr $(533 \mathrm{mPa})$. The substrate was biased with pulse DC at a frequency of 250 kHz. Pulsed power supplies (Advanced Energy Pinnacle Plus, Fort Collins, CO, USA) (100 kHz) were used to drive the $\mathrm{Si}$ target at current mode. The target current applied to the pure Si target was fixed at $3.0 \mathrm{~A}$ (sputtering power $\sim 0.7 \mathrm{~kW}$ ), corresponding to the sputtering power of $1.4 \mathrm{~kW}$. The $\mathrm{SiO}_{x}$ layer was deposited by sputtering the $\mathrm{Si}$ target in $\mathrm{Ar} / \mathrm{O}_{2}$ mixed gases, with a deposition time of $30 \mathrm{~min}$, and maintaining the oxygen flow rate at $50 \mathrm{sccm}$, and the $\mathrm{Ar} / \mathrm{O}_{2}$ pressure at $3.4 \times 10^{-3}$ Torr $(453 \mathrm{mPa})$. All the deposition was carried out at ambient temperature. During sputtering, the substrates were not heated intentionally. This method can effectively penetrate the polished surface of zirconia and deposit a silica layer around $46 \pm 2 \mu \mathrm{m}$ (Figure 1) in thickness. 


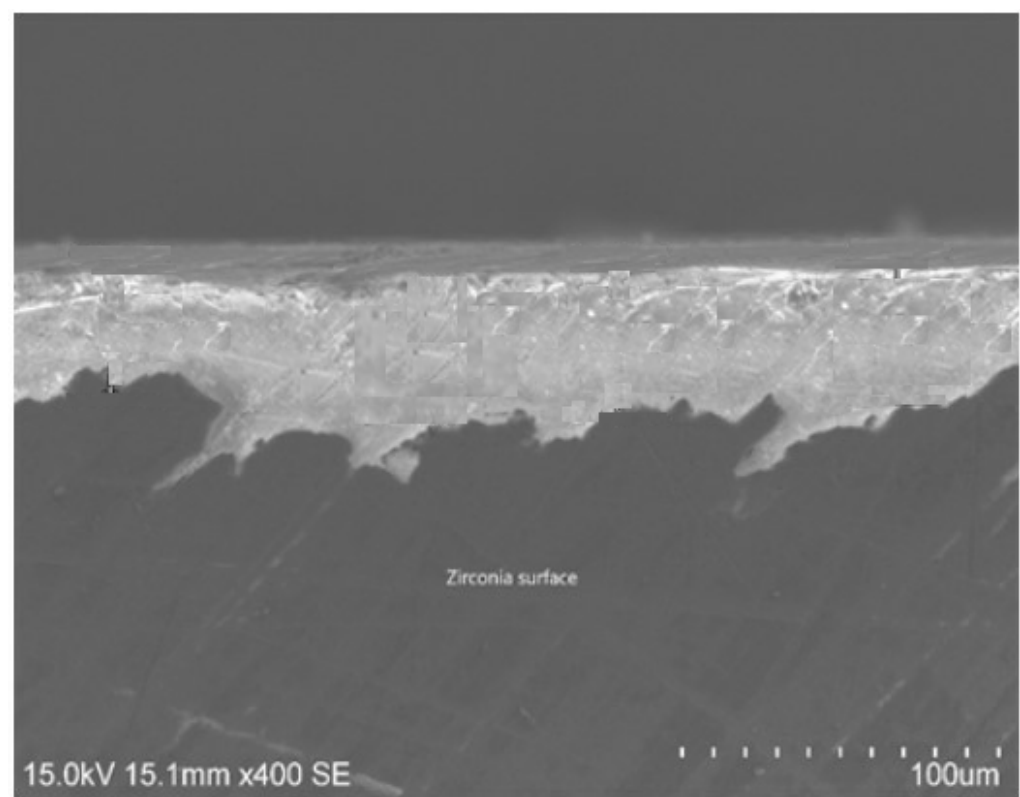

Figure 1. SEM micrograph showing the average thickness of the deposited silica layer on PVD zirconia samples.

\subsection{Preparation of the Silane Primer}

The silane primer, 1.0 vol.\% of 3-methacryloxypropyltrimethoxysilane (MPS) was prepared in a solvent mixture of 95.0 vol.\% absolute ethanol and $5.0 \mathrm{vol} . \%$ deionized water according to previous protocol [30]. Briefly, the $\mathrm{pH}$ of the solvent mixture was adjusted to 4.5 by $1 \mathrm{M}$ acetic acid. The silane solution was allowed to hydrolyze for one hour before use. The prepared silane was brushed over the coated zirconia surface with a fine brush to wet its surface prior to resin cement application and left for $5 \mathrm{~min}$ to allow for solvent evaporation and chemical reaction to take place between the silane and silica.

\subsection{Bonding of Resin Composite to Silica-Coated and Silanized Zirconia}

RelyX Unicem Aplicap (3M ESPE, Seefeld, Germany) resin composite cement was activated and mixed according to manufacturers' instructions. The mixed resin cement was dispensed carefully into a polyethylene mold (with internal diameter of $3.5 \mathrm{~mm} \times 6.0 \mathrm{~mm}$ height) which was positioned on the silica-coated and silanized zirconia surface. Finally, it was light cured for $40 \mathrm{~s}$ from top and laterally, then the mold was removed carefully after curing.

\subsection{Shear Bond Strength (SBS) Test}

The samples were mounted on a jig on a universal testing machine (ElectroPuls ${ }^{\mathrm{TM}} \mathrm{E} 3000$, Instron, Norwood, MA, USA). A constant load of $3000 \mathrm{~N}$ was applied at a cross-head speed of $1.0 \mathrm{~mm} / \mathrm{min}$ until fracture occurred. The SBS was calculated by dividing the maximum fracture loading with the circular area of the resin stub.

\subsection{Mode of Failure}

The mode of failure in zirconia specimens was assessed under the light microscope at $25 \times$ after shear bond strength testing. When the remaining resin cement stub was less than $1 / 3$ of the bonded area, the failure mode was assigned as "adhesive" and when the remaining was more than $1 / 3$ but less than $2 / 3$, it was assigned as "mixed". When the amount remaining was more than $2 / 3$, it was assigned as "cohesive" failure [31]. 


\subsection{Surface Roughness $\left(R_{a}\right)$}

The surface roughness $\left(R_{\mathrm{a}}\right)$ of the polished zirconia surface and different coatings was measured using an electro-mechanical profilometer (Surtronic 3, Taylor Hobson, Leicester, UK). Three readings were taken at random regions on the zirconia surfaces and the average was calculated.

\subsection{Characterization of Phase Transformation of Zirconia Using X-ray Diffraction (XRD)}

The tetragonal $(t)$ to monoclinic $(m)$ phase transformation of zirconia after both TSC and PVD coating processes was analyzed using XRD (Rigaku SmartLab $9 \mathrm{~kW}$, Tokyo, Japan). The scan range was set at $5^{\circ}-60^{\circ}$ and with a scan speed of $2^{\circ} / \mathrm{min}$. Patterns identification was matched using a MATCH! Phase identification software (version 3.7.1.132) (Crystal Impact, Bonn, Germany).

\subsection{Scanning Electron Microscopy (SEM) and Energy-Dispersive X-ray Spectroscopy (EDX) Analysis}

The surface topography of zirconia coated samples from each surface treatment method was evaluated by SEM (SU1510, Hitachi, Tokyo, Japan) for coating characterization, and the elemental analysis of the coating was assessed using EDX (1XRF System, Brandt Instruments, Inc., Prairieville, LA, USA).

\subsection{Statistical Analysis}

The difference in the mean shear bond strength between the test groups was analyzed using one-way ANOVA; SPSS v 25 (IBM Statistics, IBM. Chicago, IL, USA), with the shear bond strength as the dependent variable and silica coating techniques and aging conditions as the independent variables. The tukey's post hoc test and $t$-test were used to establish significance at the confidence level of $\alpha=0.05$.

\section{Results}

\subsection{Shear Bond Strength (SBS)}

The mean and standard deviation shear bond strength values of resin cement to coated zirconia surfaces for all groups are summarized in Table 1. Results show that the highest mean SBS value $(12.7 \pm 1.9 \mathrm{MPa})$ was recorded by the thermo-cycled PVD group. The mean SBS values in PVD group were statistically higher than the TSC group $(p<0.05)$. In dry storage condition, the $t$-test showed that PVD produced significantly higher SBS mean values than TSC $(p=0.006<0.05)$, but no significant difference was found between TSC and PVD groups under thermo-cycling aging condition.

Table 1. The mean and standard deviation values of the shear bond strength of TSC and PVD-coated zirconia under different aging conditions.

\begin{tabular}{clc}
\hline \multirow{2}{*}{ Groups } & Storage & $\begin{array}{c}\text { Shear Bond Strength in MPa } \\
\text { (Mean } \pm \text { SD) }\end{array}$ \\
\hline \multirow{2}{*}{ TSC } & Dry storage & $10.2 \pm 1.2^{\mathrm{a} / \mathrm{I} / \alpha}$ \\
& Thermo-cycled & $12.0 \pm 1.0^{\mathrm{b} / \mathrm{i} / \alpha \beta}$ \\
\hline \multirow{2}{*}{ PVD } & \multicolumn{1}{c}{ Dry storage } & $11.7 \pm 1.3^{\mathrm{A} / \mathrm{II} / \alpha \beta}$ \\
& \multicolumn{1}{c}{ Thermo-cycled } & $12.7 \pm 1.9^{\mathrm{A} / \mathrm{i} / \beta}$ \\
\hline \multirow{2}{*}{ Level 1 } & Capital and small letters alphabets indicate significant difference within test groups-TSC and PVD \\
& (Independent $t$-test); & \\
\hline \multirow{2}{*}{ Level 2 } & Capital and small roman numerals indicate significant differences between the storage \\
& conditions-Dry and Thermo-cycled (Independent t-test); \\
\hline \multirow{2}{*}{ Level 3 } & $\begin{array}{l}\text { Greek alphabets indicate significant differences between the groups overall (One-way ANOVA } \\
\text { with the Tukey's HSD post hoc test); } p<0.05 \text { is significant. }\end{array}$ \\
\hline
\end{tabular}




\subsection{EDX Elemental Analysis of Treated Surfaces}

The elemental analysis of coated zirconia samples is shown in Figure 2. In TSC, the elemental analysis revealed the existence of $\mathrm{Zr}, \mathrm{Si}, \mathrm{O}$, and $\mathrm{Al}$. For the PVD coating, the elemental analysis showed high amount of $\mathrm{Si}$ in addition to $\mathrm{Zr}, \mathrm{O}$, and $\mathrm{Ar}$ (Table 2).

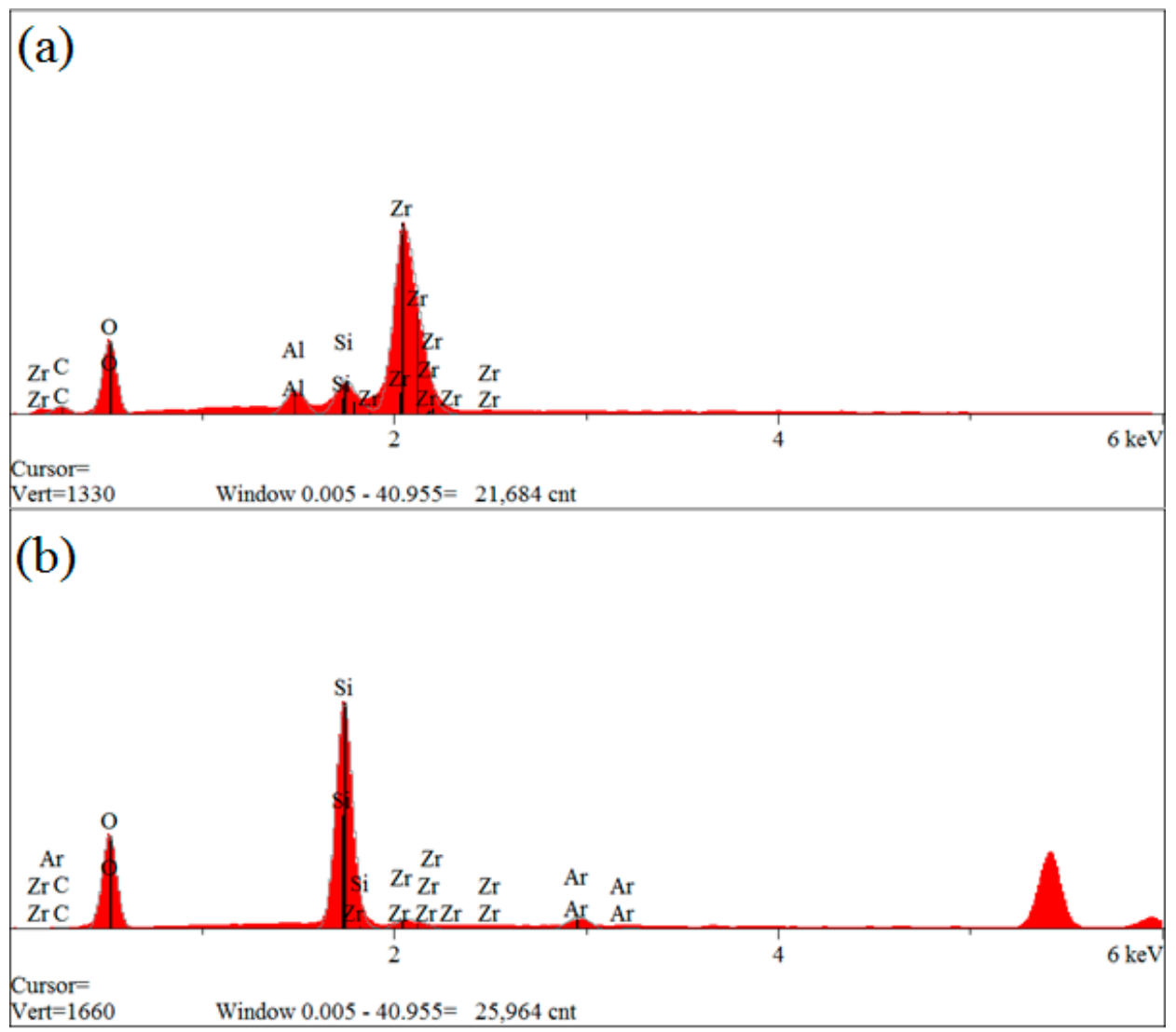

Figure 2. The EDX analysis of and (a) TSC and (b) PVD-coated zirconia surfaces.

Table 2. EDX elemental analysis of PVD and TSC coatings.

\begin{tabular}{ccc}
\hline Element & TSC (at.\%) & PVD (at.\%) \\
\hline $\mathrm{C}$ & 14.691 & 20.437 \\
$\mathrm{O}$ & 48.122 & 17.563 \\
$\mathrm{Al}$ & 5.336 & 0 \\
$\mathrm{Si}$ & 5.462 & 51.853 \\
$\mathrm{Zr}$ & 26.389 & 4.656 \\
$\mathrm{Ar}$ & 0 & 5.491 \\
\hline
\end{tabular}

\subsection{Mode of Failure Assessment}

Figure 3 shows representative micrographs of debonded specimens. The modes of failure of all tested specimens are shown in Figure 4 . Results suggest that the predominant failure mode was adhesive failure, $60 \%$, among all the specimens, followed by mixed failure $30 \%$. Some cohesive failures $10 \%$ were also observed. 

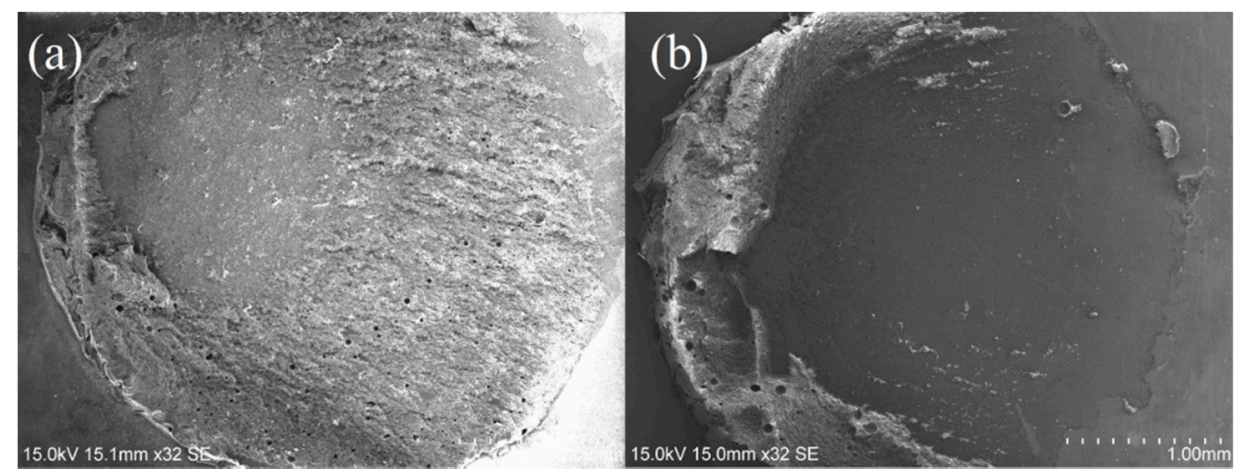

Figure 3. SEM micrographs representing the debonded areas of mixed failure on (a) TSC and adhesive failure on (b) PVD-coated zirconia samples at $32 \times$ magnification.

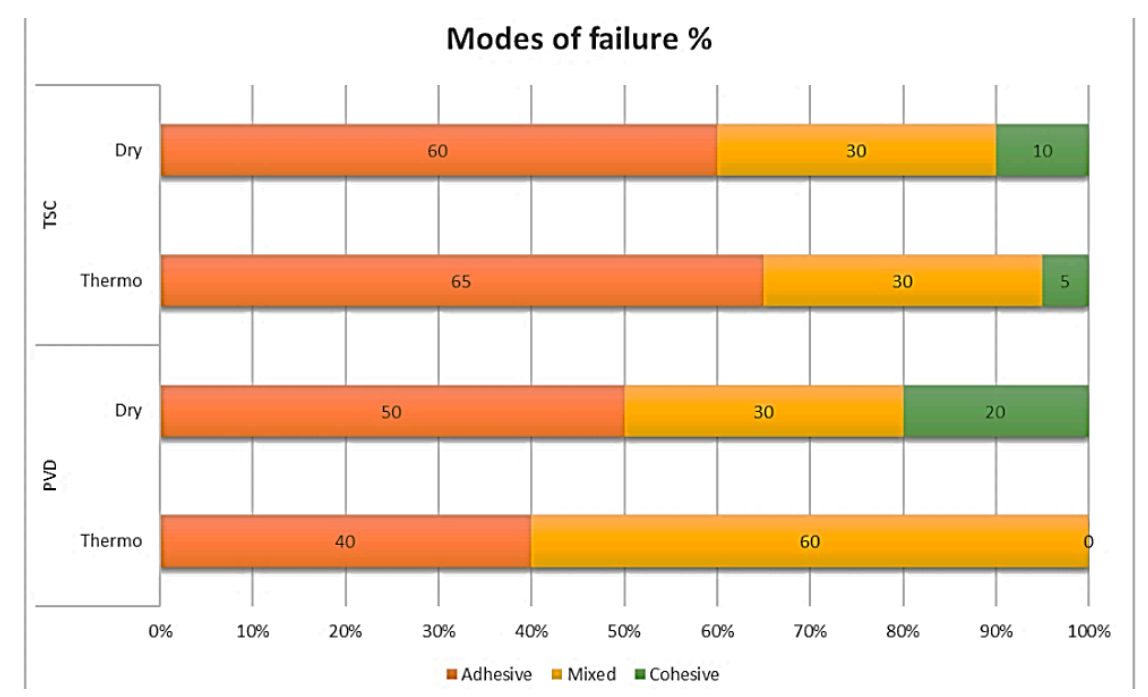

Figure 4. Different modes of failure of all tested zirconia samples.

\subsection{Surface Roughness}

The mean $\left(R_{\mathrm{a}}\right)$ of polished zirconia surface, TSC and PVD-coated zirconia are listed in Figure 5. No difference was found in the mean surface roughness between polished and PVD-coated zirconia. The tukey's post hoc test showed that there was a significant difference between TSC and both polished and PVD-coated zirconia $(p=0.000019<0.05)$.

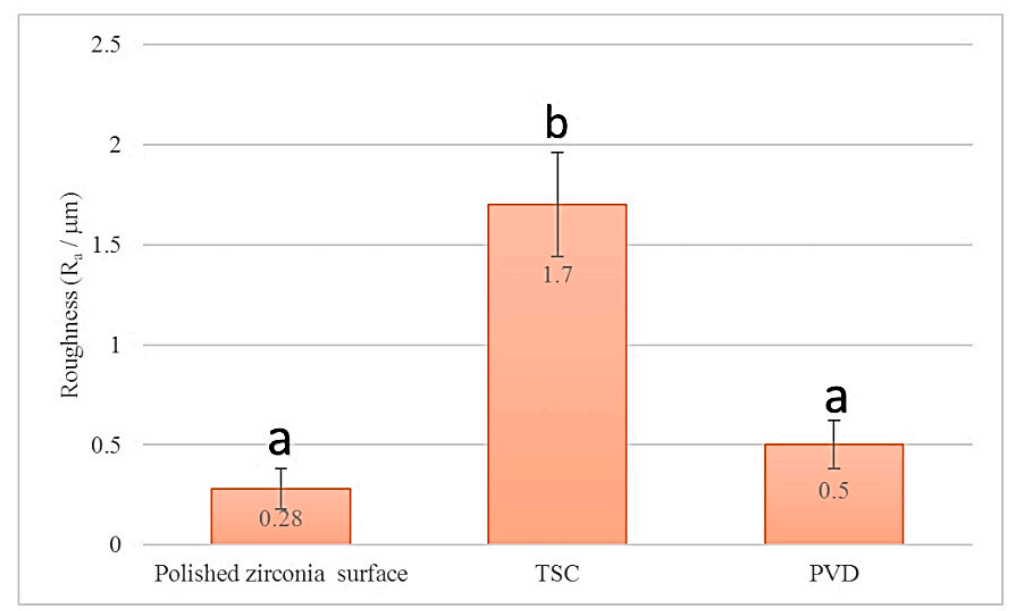

Figure 5. The mean surface roughness $\left(R_{\mathrm{a}}\right)$ values of polished, TSC and PVD-coated zirconia samples. Different lowercase letters indicate that there is a significant difference $p<0.001$. 


\subsection{XRD Analysis}

Results of XRD phase analysis (Figure 6) showed that there was a $t \rightarrow m$ phase transformation in TSC zirconia samples. Intense peak of $m$-phase was detected at $31.5^{\circ} 2 \theta$, while in PVD samples, no m-phase was detected.

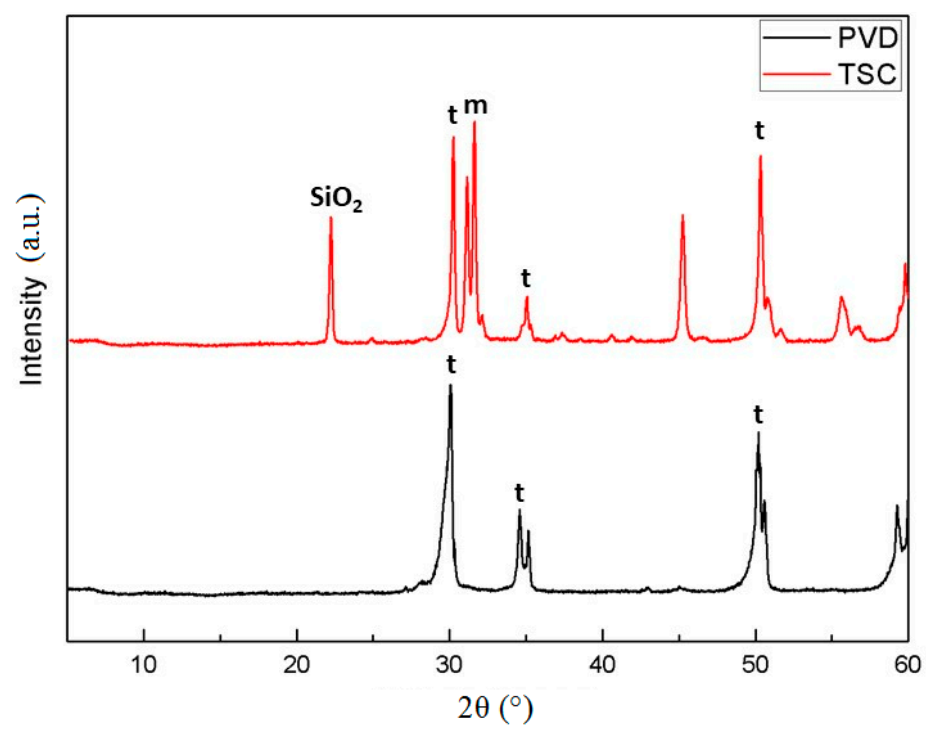

Figure 6. XRD patterns of TSC and PVD. No $t \rightarrow m$ phase transformation was detected in PVD-coated zirconia sample.

\subsection{Surface Morphology Analysis}

SEM micrographs of polished zirconia surface, TSC and PVD-coated zirconia surfaces are shown in Figure 7. From the micrographs, polished zirconia surface showed a smooth surface topography, while the TSC coated zirconia surface was irregular. The PVD-coated surface has shown a uniform silica film on zirconia surface.

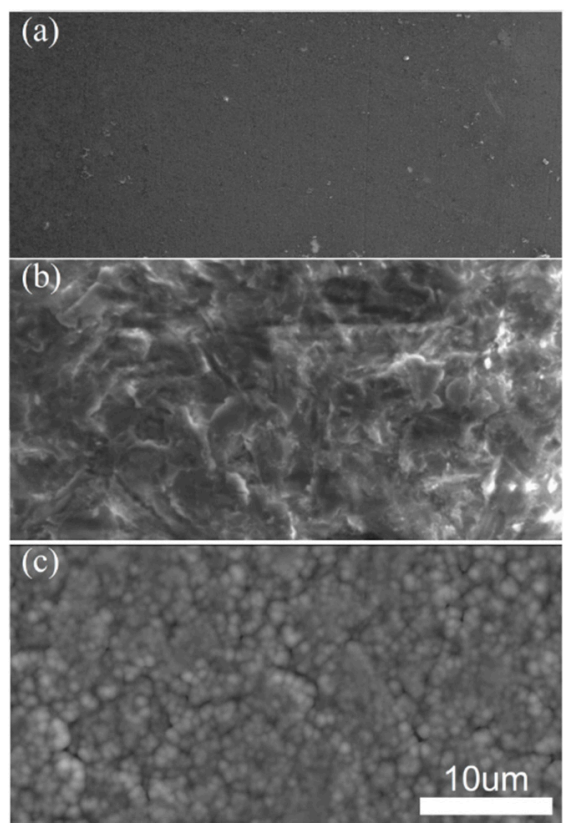

Figure 7. SEM micrographs showed smooth surface topography on (a) polished zirconia surface, typical irregular surface topography on (b) TSC zirconia surface, and regular silica particles morphology on (c) PVD-coated zirconia. 


\section{Discussion}

In the current study, a thin silica coating (around $46 \mu \mathrm{m}$ ) using physical vapor deposition (PVD) with high ionization sputtering (Argon arc splitting) processing technology was successfully applied onto dental zirconia. An improved resin-zirconia SBS (Table 1), achieved alongside a very high silica content (51.853 at.\% Si, Table 2), no $t \rightarrow m$ transformation (Figure 4), and no surface roughening (Figure 5), was detected. The improved SBS may be explained by the high silica content in the deposited layer, which has led to more hydrolytically stable-Si-O-Si- linkages at the bonding interface with the silane primer [21].

Although Queiroz et al. [25] reported high SBS bond strength values when $\mathrm{SiO}_{x}$ film was deposited on zirconia using magnetron sputtering (they also found that this coating method improved bond strength without compromising the quality of the substrate surface), they did not study the effect of thermal aging effects on bond durability. It should be pointed out that the film thickness and silica content of the deposited layer were different from our current study. Indeed, this study utilized a controlled PVD sputtering process, which adopts electric arc discharge technology with low voltage and high current performed under vacuum conditions, resulting in a vacuum-sealing filming with no contamination. This PVD solution can easily acquire coatings with super hardness and wear-resistance, which other solutions can hardly achieve.

TSC was used as the control group, since it is a widely-used protocol for surface treatment of zirconia, and it is also the conventional conditioning pretreatment technique carried out by dental laboratories [28]. Furthermore, we compared TSC with PVD, because both are based on silica-coating. The Si content found on zirconia surface is only 5.462 at.\% (Table 2), i.e., 10-fold less than PVD. These results are in accordance with previously reported results [16,32]. In fact, the silica-coated alumina particles are able to embed into the zirconia surface to form a silica layer and also increase the surface roughness for micromechanical interlocking. Thus, the adhesion strength from TSC is contributed by both mechanical and chemical bonding. It is important to notice that the silica content is a very important factor using silane as a ceramic primer, because the formation of -Si-O-Si- is vital for any chemical bonding. As previously reported [24], ultrasonic cleaning during TSC procedures can result in detachment of some silica particles, which negatively affects the silica content on the zirconia substrate and hence a decrease in bond durability. On the other hand, ideally, the deposition of silica coating for silane bonding should not induce any phase transformation or create any cracks on the zirconia surface; otherwise, this could affect the long-term performance of zirconia [33].

Our XRD findings suggested that $t \rightarrow m$ transformation was significantly found (Figure 4 ) in TSC, which is prone to form micro-cracks, but not in PVD. In fact, TSC is a technique that uses abrasive grit particles (here, silica-coated alumina) to attack the material surface and generate a swarf-like surface. As a result, a rough surface in terms of micro-scale is created and silica layer is deposited by embedment of grits. The deposited silica layer did not create any stresses or induce any phase transformation, but such an attack of grits on sintered zirconia cracked the surface zirconia grains, and the $t$ phase grains transformed itself to $m$ phase (larger in grain size) in order to increase crack length, i.e., a toughening mechanism, to prevent it from cracking. Ultimately, $t \rightarrow m$ transformation was found in the TSC-treated surface [23]. On the other hand, PVD utilized a sputtering technique to coat Si directly on the zirconia, which did not cause any stress on or induce any phase transformation on zirconia. As a result, TSC might not be the best solution to enhance bond strength as it also induces subsurface damage in zirconia.

Laboratory tests for measuring the bond strength give approximate values for the real clinical conditions. The shear bond strength test, as one of the adhesion strength testing methods, is widely used because of test simplicity and ease of sample preparation and loading over the testing machine [34]. The limitations of this test method are that the shear bond strength varies with sample shape and test configurations [35], in addition to the development of nonhomogeneous stress distribution at the bonding interface [36,37]. Nevertheless, the high crystallinity and toughness of zirconia makes it difficult to prepare micro tensile bond strength samples. Furthermore, cutting the sintered zirconia 
specimen into a micro-tensile bond strength sample might involve heat and water, which may induce surface flaws, grains enlargement [38] and possibly $t \rightarrow m$ transformation. As such, other test methods such as flexural bond strength test [12] that have higher sensitivity could be utilized. Further studies are necessary as these tests are rarely performed.

In the current study, we used a freshly prepared experimental (MPS) silane primer. It was reported that freshly prepared experimental MPS performs better than pre-hydrolyzed commercial forms [39]. Thermo-cycling is considered one of the common laboratory artificial aging methods used in assessing bond durability and stability. It simulates both thermal changes and water aging conditions that occur in the oral environments fairly well [40]. The SBS increased after thermo-cycling in both surface treatments; this might be attributed to further post-polymerization of residual monomers, accelerated by a dwelling period at the relatively high temperature of $55{ }^{\circ} \mathrm{C}$ [39]. This is in agreement with Matinlinna and Lassila [39], who reported that the SBS increased after thermo-cycling. However, our current results are in disagreement with Lee et al. [41], who found that bond strength decreased after thermo-cycling, which may be due to the different surface treatments, resin composite, and silane coupling agent used in our study.

From the mode of failure analysis (Figure 4), adhesive failure was the most prevalent, with some mixed and few cohesive failures. The mode of failure at the bonding interface gives an indication of the bond strength of the adhesive to the substrate [41]. If the resin bond is strong enough, failure will occur within the substrate. The adhesive failure might be due to the detachment of resin composite from the interface layer or silica-coating from the zirconia surface [31]. These results are in agreement with Cheng et al. [31]. This suggests that the bond between the resin and zirconia was weaker than the resin substrate. Based on this, a finite-element study [37] might be useful in order to theoretically predict the failure phenomena of resin adhesion on TSC and PVD-coated zirconia.

The surface roughness of TSC was higher than that of PVD, which was a result of the sandblasting with silica-coated alumina particles. The low surface roughness results of the PVD-coated zirconia indicate that this surface treatment method maintains a smooth zirconia surface. SEM was used to observe the morphological changes that might occur on zirconia after surface treatment with both TSC and PVD. SEM micrographs have shown that the TSC-coated zirconia surface was irregular, which was as a result of the sandblasting process, as reported by Kulunk et al. [42]. The PVD-coated surface showed uniform silica coating on the zirconia surface; accordingly, the marginal adaptation of zirconia is expected to not be compromised [43]. This smoothness could even result in reduced bacterial accumulation on the treated zirconia surface than TSC ones [44].

The null hypothesis of the current study was rejected, as the resin-zirconia SBS after PVD coating was higher than TSC, and thermo-cycling did not deteriorate bond strength. Further studies are recommended to evaluate the long-term durability of the PVD coating and to assess the effect of different $\mathrm{Si} / \mathrm{O}$ ratios on bond strength. It is also recommended to compare PVD coating with air-abrasion with $50 \mu \mathrm{m}$ alumina particles at $0.25 \mathrm{MPa}$, followed by 10-methacryloxydecyl-dihydrogenphosphate (MDP) primer, as the recommended first choice of bonding to zirconia [20]. The limitations of the PVD coating method are that it requires specific machine equipment and the cost of the procedures is high.

\section{Conclusions}

It can be concluded that reliable bonding to zirconia could be achieved with chemical bonding only without the need for any micromechanical roughening methods such as air particle abrasion with alumina or TSC, which affect the structural properties of zirconia substrate. PVD coating by deploying high silica content is a promising method that improves resin-zirconia bond strength and overcomes the disadvantages of TSC. Thermal aging conditions did not deteriorate bond strength in both TSC and PVD. The amount of silica content in the deposited coating plays an important role in resin-zirconia bonding with the application of a silane coupling agent. 
Author Contributions: Conceptualization, M.M.A. and J.P.M.; Methodology, M.M.A., C.Y.K.L., J.K.H.T. and J.P.M.; Formal Analysis, M.M.A. and J.K.H.T.; Investigation, M.M.A.; Resources, J.K.H.T. and J.P.M.; Data Curation, M.M.A. and J.K.H.T.; Writing-Original Draft Preparation, M.M.A.; Writing-Review and Editing, C.Y.K.L., J.K.H.T. and J.P.M.; Supervision, J.K.H.T.; Project Administration, J.P.M.

Funding: This research received no external funding.

Acknowledgments: We would like to thank Paul Lee, the technician, for his assistance in the shear bond strength test measurement and operation of the thermocycling machine. We would also like to thank Bobby Li, the technical support of the Techmart Industrial Company, for his assistance to prepare the silica-coated samples by PVD method.

Conflicts of Interest: The authors declare no conflict of interest.

\section{References}

1. Piascik, J.R.; Swift, E.J.; Thompson, J.Y.; Grego, S.; Stoner, B.R. Surface modification for enhanced silanation of zirconia ceramics. Dent. Mater. 2009, 25, 1116-1121. [CrossRef] [PubMed]

2. Blatz, M.B.; Sadan, A.; Kern, M. Resin-ceramic bonding: A review of the literature. J. Prosthet. Dent. 2003, 89, 268-274. [CrossRef] [PubMed]

3. Della Bona, A.; Pecho, O.E.; Alessandretti, R. Zirconia as a dental biomaterial. Materials 2015, 8, 4978-4991. [CrossRef] [PubMed]

4. Pittayachawan, P.; McDonald, A.; Petrie, A.; Knowles, J.C. The biaxial flexural strength and fatigue property of Lava Y-TZP dental ceramic. Dent. Mater. 2007, 23, 1018-1029. [CrossRef] [PubMed]

5. Denry, I.; Kelly, J.R. State of the art of zirconia for dental applications. Dent. Mater. 2008, 24, $299-307$. [CrossRef] [PubMed]

6. Hannink, R.H.J.; Kelly, P.M.; Muddle, B.C. Transformation toughening in zirconia-containing ceramics. J. Am. Ceram. Soc. 2000, 83, 461-487. [CrossRef]

7. Borges, G.A.; Sophr, A.M.; de Goes, M.F.; Sobrinho, L.C.; Chan, D.C. Effect of etching and airborne particle abrasion on the microstructure of different dental ceramics. J. Prosthet. Dent. 2003, 89, 479-488. [CrossRef]

8. Soares, C.J.; Soares, P.V.; Pereira, J.C.; Fonseca, R.B. Surface treatment protocols in the cementation process of ceramic and laboratory-processed composite restorations: A literature review. J. Esthet. Restor. Dent. 2005, 17, 224-235. [CrossRef] [PubMed]

9. Tinschert, J.; Schulze, K.A.; Natt, G.; Latzke, P.; Heussen, N.; Spiekermann, H. Clinical behavior of zirconia-based fixed partial dentures made of DC-Zirkon: 3-year results. Int. J. Prosthodont. 2008, 21, 217-222. [PubMed]

10. Chen, J.H.; Matsumura, H.; Atsuta, M. Effect of different etching periods on the bond strength of a composite resin to a machinable porcelain. J. Dent. 1998, 26, 53-58. [CrossRef]

11. Tian, T.; Tsoi, J.K.; Matinlinna, J.P.; Burrow, M.F. Aspects of bonding between resin luting cements and glass ceramic materials. Dent. Mater. 2014, 30, e147-e162. [CrossRef] [PubMed]

12. Wong, A.C.H.; Tian, T.; Tsoi, J.K.H.; Burrow, M.F.; Matinlinna, J.P. Aspects of adhesion tests on resin-glass ceramic bonding. Dent. Mater. 2017, 33, 1045-1055. [CrossRef] [PubMed]

13. Liu, D.; Tsoi, J.K.; Matinlinna, J.P.; Wong, H.M. Effects of some chemical surface modifications on resin zirconia adhesion. J. Mech. Behav. Biomed. Mater. 2015, 46, 23-30. [CrossRef] [PubMed]

14. Kitayama, S.; Nikaido, T.; Maruoka, R.; Zhu, L.; Ikeda, M.; Watanabe, A.; Foxton, R.M.; Miura, H.; Tagami, J. Effect of an internal coating technique on tensile bond strengths of resin cements to zirconia ceramics. Dent. Mater. J. 2009, 28, 446-453. [CrossRef] [PubMed]

15. Kern, M.; Wegner, S.M. Bonding to zirconia ceramic: Adhesion methods and their durability. Dent. Mater. 1998, 14, 64-71. [CrossRef]

16. Liu, D.; Matinlinna, J.P.; Tsoi, J.K.; Pow, E.H.; Miyazaki, T.; Shibata, Y.; Kan, C.W. A new modified laser pretreatment for porcelain zirconia bonding. Dent. Mater. 2013, 29, 559-565. [CrossRef] [PubMed]

17. Liu, D.; Pow, E.H.N.; Tsoi, J.K.; Matinlinna, J.P. Evaluation of four surface coating treatments for resin to zirconia bonding. J. Mech. Behav. Biomed. Mater. 2014, 32, 300-309. [CrossRef] [PubMed]

18. Aboushelib, M.N.; Kleverlaan, C.J.; Feilzer, A.J. Selective infiltration-etching technique for a strong and durable bond of resin cements to zirconia-based materials. J. Prosthet. Dent. 2007, 98, 379-388. [CrossRef]

19. Wolfart, M.; Lehmann, F.; Wolfart, S.; Kern, M. Durability of the resin bond strength to zirconia ceramic after using different surface conditioning methods. Dent. Mater. 2007, 23, 45-50. [CrossRef] [PubMed] 
20. Kern, M. Bonding to oxide ceramics—Laboratory testing versus clinical outcome. Dent. Mater. 2015, 31, 8-14. [CrossRef] [PubMed]

21. Matinlinna, J.P.; Areva, S.; Lassila, L.V.; Vallittu, P.K. Characterization of siloxane films on titanium substrate derived from three aminosilanes. Surf. Interface Anal. 2004, 36, 1314-1322. [CrossRef]

22. Lung, C.Y.; Matinlinna, J.P. Aspects of silane coupling agents and surface conditioning in dentistry: An overview. Dent. Mater. 2012, 28, 467-477. [CrossRef] [PubMed]

23. Zhang, Y.; Lawn, B.R. Novel zirconia materials in dentistry. J. Dent. Res. 2018, 97, 140-147. [CrossRef] [PubMed]

24. Nishigawa, G.; Maruo, Y.; Irie, M.; Oka, M.; Yoshihara, K.; Minagi, S.; Nagaoka, N.; Yoshida, Y.; Suzuki, K. Ultrasonic cleaning of silica-coated zirconia influences bond strength between zirconia and resin luting material. Dent. Mater. J. 2008, 27, 842-848. [CrossRef] [PubMed]

25. Queiroz, J.R.C.; Duarte, D.A.; Souza, R.O.A.; Fissmer, S.F.; Massi, M.; Bottino, M.A. Deposition of $\mathrm{SiO}_{x}$ thin films on Y-TZP by reactive magnetron sputtering: Influence of plasma parameters on the adhesion properties between Y-TZP and resin cement for application in dental prosthesis. Mater. Res. 2011, 14, 212-216. [CrossRef]

26. Sesthan, K.; Schepis, D. Handbook of Thin Film Deposition, 4th ed.; Elsevier: Cambridge, MA, USA, 2018.

27. Cakir-Omur, T.; Gozneli, R.; Ozkan, Y. Effects of silica coating by physical vapor deposition and repeated firing on the low-temperature degradation and flexural strength of a zirconia ceramic. J. Prosthodont. 2019, 28, e186-e194. [CrossRef] [PubMed]

28. Matinlinna, J.P.; Lassila, L.V.; Vallittu, P.K. Pilot evaluation of resin composite cement adhesion to zirconia using a novel silane system. Acta Odontol. Scand. 2007, 65, 44-51. [CrossRef] [PubMed]

29. Ho, B.J.; Tsoi, J.K.; Liu, D.; Lung, C.Y.; Wong, H.M.; Matinlinna, J.P. Effects of sandblasting distance and angles on resin cement bonding to zirconia and titanium. Int. J. Adhes. Adhes. 2015, 62, 25-31. [CrossRef]

30. Matinlinna, J.P.; Lassila, L.V.; Vallittu, P.K. The effect of five silane coupling agents on the bond strength of a luting cement to a silica-coated titanium. Dent. Mater. 2007, 23, 1173-1180. [CrossRef] [PubMed]

31. Cheng, H.C.; Tsoi, J.H.; Zwahlen, R.A.; Matinlinna, J.P. Effects of silica-coating and a zirconate coupling agent on shear bond strength of flowable resin-zirconia bonding. Int J. Adhes. Adhes. 2014, 50, 11-16. [CrossRef]

32. Matinlinna, J.P.; Heikkinen, T.; Ozcan, M.; Lassila, L.V.; Vallittu, P.K. Evaluation of resin adhesion to zirconia ceramic using some organosilanes. Dent. Mater. 2006, 22, 824-831. [CrossRef] [PubMed]

33. Atsu, S.S.; Kilicarslan, M.A.; Kucukesmen, H.C.; Aka, P.S. Effect of zirconium-oxide ceramic surface treatments on the bond strength to adhesive resin. J. Prosthet. Dent. 2006, 95, 430-436. [CrossRef] [PubMed]

34. Zhang, Y.; Lawn, B.; Rekow, E.D.; Thompson, V.P. Effect of sandblasting on the long-term performance of dental ceramics. J. Biomed. Mater. Res. Part B Appl. Biomater. 2004, 71, 381-386. [CrossRef] [PubMed]

35. Rasmussen, S.T. Analysis of dental shear bond strength tests, shear or tensile? Int. J. Adhes. Adhes. 1996, 16, 147-154. [CrossRef]

36. Placido, E.; Meira, J.B.; Lima, R.G.; Muench, A.; de Souza, R.M.; Ballester, R.Y. Shear versus micro-shear bond strength test: A finite element stress analysis. Dent. Mater. 2007, 23, 1086-1092. [CrossRef] [PubMed]

37. Jin, X.Z.; Homaei, E.; Matinlinna, J.P.; Tsoi, J.K. A new concept and finite-element study on dental bond strength tests. Dent. Mater. 2016, 32, e238-e250. [CrossRef] [PubMed]

38. Ho, C.M.; Ding, H.; Chen, X.; Tsoi, J.K.; Botelho, M.G. The effects of dry and wet grinding on the strength of dental zirconia. Ceram. Int. 2018, 44, 10451-10462. [CrossRef]

39. Matinlinna, J.P.; Lassila, L.V. Enhanced resin-composite bonding to zirconia framework after pretreatment with selected silane monomers. Dent. Mater. 2011, 27, 273-280. [CrossRef] [PubMed]

40. Lung, C.Y.; Liu, D.; Matinlinna, J.P. Surface treatment of titanium by a polydimethylsiloxane coating on bond strength of resin to titanium. J. Mech. Behav. Biomed. Mater. 2015, 41, 168-176. [CrossRef] [PubMed]

41. Lee, J.J.; Kang, C.K.; Oh, J.W.; Seo, J.M.; Park, J.M. Evaluation of shear bond strength between dual cure resin cement and zirconia ceramic after thermocycling treatment. J. Adv. Prosthodont. 2015, 7, 1-7. [CrossRef] [PubMed]

42. Külünk, S.; Külünk, T.; Ural, C.; Kurt, M.; Baba, S. Effect of air abrasion particles on the bond strength of adhesive resin cement to zirconia core. Acta Odontol. Scand. 2011, 69, 88-94. [CrossRef] [PubMed] 
43. Vanderlei, A.; Bottino, M.A.; Valandro, L.F. Evaluation of resin bond strength to yttria-stabilized tetragonal zirconia and framework marginal fit: Comparison of different surface conditionings. Oper. Dent. 2014, 39, 50-63. [CrossRef] [PubMed]

44. Han, A.; Tsoi, J.K.H.; Matinlinna, J.P.; Chen, Z. Influence of grit-blasting and hydrofluoric acid etching treatment on surface characteristics and biofilm formation on zirconia. Coatings 2017, 7, 130. [CrossRef]

(C) 2019 by the authors. Licensee MDPI, Basel, Switzerland. This article is an open access article distributed under the terms and conditions of the Creative Commons Attribution (CC BY) license (http://creativecommons.org/licenses/by/4.0/). 